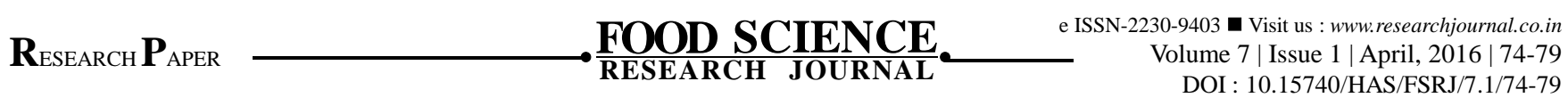

\title{
Impact of nutrition education on iron deficiency anaemia among college student of Banasthali University, Rajasthan
}

\author{
Jyoti Kumari and Ritu Prakash Dubey
}

\begin{abstract}
Malnutrition encompasses a broad spectrum of deficiencies, of which the most devastating is the deficiency of one or more of the three micronutrients namely iron, vitamin A and iodine. Anemia is a condition where oxygen carrying capacity of the blood is reduced due to reduced hemoglobin concentration in the blood. Among adolescent girls, constitute a vulnerable group, particularly in developing countries due to poverty, inadequate diet, certain disease, pregnancy / lactation and poor access to health service. Nutrition education is an instructional method that promotes healthy behaviors by imparting information that individual can use to make informed decision about food, dietary habit and health. A recent study has shown the impact of Nutrition Education on the nutritional knowledge of adolescent girls. The objective was to study the prevalence of anemia among adolescent girls and to assess the dietary awareness and dietary practices of adolescent girls. Sixty girls were selected by random Sampling. The knowledge level and Practice level pre-questionnaire was prepared to collect background information on the subjects. A dietary assessment was an essential part and was done by an oral questionnaire ( 24 hours dietary recall). The subjects were introduced to nutrition and anemia after filling in the questionnaire. They used educational materials such as posters, charts, pamphlets, models, flash cards and leaflets. The same structured questionnaire was used to test nutritional knowledge level after the educational program. A significant difference $(\mathrm{P}>0.005)$ was observed in the score of pre and post test of Nutrition Education intervention. Hence Nutrition Education is an appropriate, effective and sustainable approach to combat iron deficiency anemia.
\end{abstract}

Key Words : Anemia, Nutrition education, 24 hours dietary recall

How to cite this article : Kumari, Jyoti and Dubey, Ritu Prakash (2016). Impact of nutrition education on iron deficiency anaemia among college student of Banasthali University, Rajasthan. Food Sci. Res. J., 7(1): 74-79. 